\title{
Per Operative Discovery of Placenta Percreta and Uterine Rupture at 37 Weeks of Gestation - A Case Report
}

\author{
RAHAT AFZA CHOWDHURY ${ }^{1}$, FERDOUSI CHOWDHURY ${ }^{2}$, S. FEROZ ${ }^{3}$
}

\begin{abstract}
:
The aim is to publish this case report of rarely occurring and life threatening condition, placenta percreta causing uterine rupture at 37 weeks of gestation.

A second gravida with unevenful pregnancy was admitted in a tertiary hospital at her 37 weeks of gestation with lower abdominal pain and less foetal movement with history of one caesarean section.After admission she complaint of dull pain in whole abdomen which was gradually increasing in intensity, associated with hardening of uterus, initiallyher pulse and BP was stable, but later she had tachycardia and fetal heart rate was irregular.An emergency cesarean section was done. Immediately after opening the peritoneal cavity a perforation in uterine wall by placenta was found through which active bleeding was present.After holding the bleeding point, an incision in upper segment of uterine wall was given and an asphyxiated male baby was delivered by breech extraction. Then in presence of a senior gynaecologist caesarean hysterectomy was done keeping placenta in situ. Post operative period was uneventful for the mother but the baby was managed in neonatal care unit. Both of them were well during discharge. Placenta percreta is a rare pathological entity with challenging diagnostic and therapeutic requirement especially for resource limited settings.
\end{abstract}

Key words: placenta percreta, risk factor, caesarean hysterectomy.

Introduction:

Morbidly adherent placenta (MAP) is the abnormal adherence of placenta either wholly or partially to the underlying uterine wall. It is a life -threatening complication of pregnancy as there is considerable morbidity and mortality associated with this condition due to its potential severe haemorrhage at the time of delivery ${ }^{1,2}$.MAP is classified according to degree of penetration of chorionic villi and by the area of placental involvement into 3 types: a)Placenta accreta, b) Placenta increta, c)Placenta percreta.Placenta accreta refers to a placenta where invasion of decidua basalis and superficial penetration to myometrium is present; Placenta increta refers to a placenta that partially invades the myometrium ; Placenta percreta refers to a full thickness penetration of the myometrium and serosa by placenta with occasional invasion of adjacent structures such as bladder, pelvic peritoneum and bowel. Various case reports of uterine rupture due to placenta accreta are available. ${ }^{3,4}$ According to
ACOG it's incidence is $1: 2500$ deliveries. ${ }^{5}$ Antenatal diagnosis is required to plan delivery and minimize complications. Ultrasonogram/MRI are useful in establishing antenatal diagnosis yet. ${ }^{6}$ The incidence of abnormal placentation has been on the increase and the rising rates of cesarean section remains the major contributing factor. ${ }^{7}$ Women with two or more prior cesarean deliveries with anterior or central placenta praevia are at $40 \%$ increased risk of placenta accreta. $^{5}$

\section{Case Report :}

Mrs. Lovely a 28 years old housewife was admitted in our hospital on 16.7.2013 at 8:00pm and diagnosed as a case of $2^{\text {nd }}$ gravida 37 weeks of gestation with history of one caesarean section .She was admitted with the complaint of lower abdominal pain with less fetal movement for 1 day. The patient also complaint that she had dull pain in lower abdomen and was not associated with hardening of the uterus. On admission-

1. Assistant professor, Dept of Obys and Gynae, Ad-din Medical College and Hospital,Dhaka.

2. Associate professor and Head, Dept of Obs and Gynae, Ad-din medical College and Hospital,Dhaka.

3. Medical Officer, OSD, DGHS, Mohakhali. 


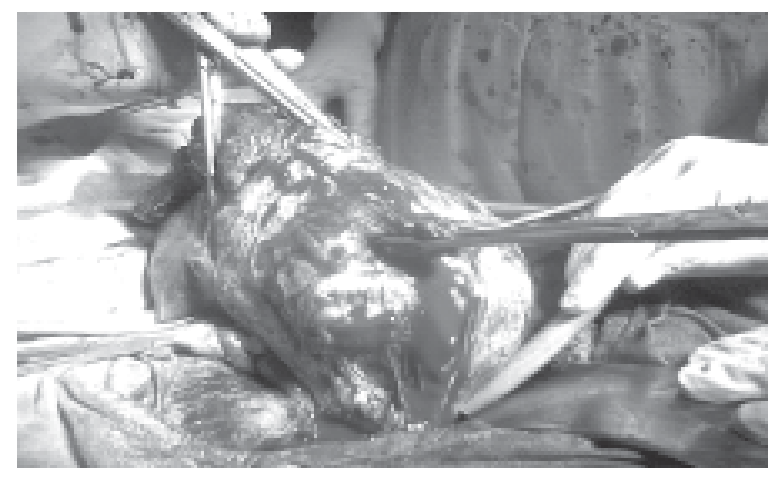

Fig.-1: Per operative picture showing perforating site of uterus by placenta being held with sponge holding forcep

Her pulse was 88 beat/min,Blood pressure was 110/ $70 \mathrm{~mm}$ of $\mathrm{Hg}$. She was mildly anaemic. On per abdominal examination-uterus was non-tender, scar tenderness was absent and fetal heart rate was 142 beat/min,regular. On per vaginal examination: cervixsoft, os-closed,there was no bleeding or discharge. Her investigation revealed -

Blood group- B +ve; Hb-11.0gm/dl, Total platelet count 200000/cumm.

Urine $\mathrm{R} / \mathrm{M} / \mathrm{E}$ report revealed - Albumin-nil;Pus cell2-5/HPF; epithelial cell -2-5/HPF.

USG of pregnancy profile reveals- A case of 36 weeks single alive pregnancy, with fetal weight- $2.6 \mathrm{~kg}$, liqouradequate, placenta -anterior and away from os.

Later on (at 10:00pm) she developed pain in whole abdomen, which was gradually increasing in intensity and associated with hardening of uterus. Her pulsewas -110 beat/min, BP was 90/60 mm of $\mathrm{Hg}$. Fetal heart rate was irregular.An emergency caesarean section was performed. Immediately after opening of the

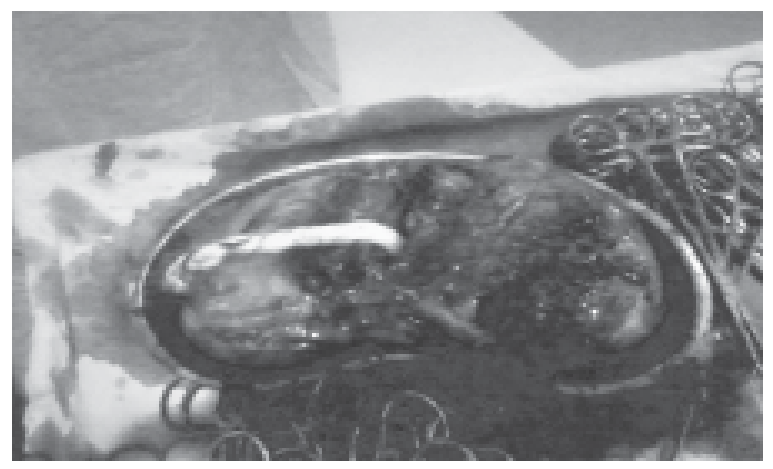

Fig.-2: Resected uterus with placenta in situ after cesarean hysterectomy abdomen it was found that abdomen was full of blood and a perforation was seen on the anterior wall of the lower part of the uterus from where bleeding was continued. Placenta was extending throughout the anterior wall up-to serosa. The perforating site was hold with sponge holding forcep. Then an incision was given on the upper segment of the uterus and the baby was delivered by breech extraction. No attempt was made to pull the placenta. Then quick decision was taken for caesarean hysterectomy and caesarean hysterectomy was done with caution.Per operative blood loss could not be estimated but 4 unit of fresh blood was transfused to maintain haemodynamic stability. Her postoperative period was uneventful. The patient was discharged on 7th post operative day.

Histopathological report shows sections of whole thickness of uterine wall with chorionic villi infiltrating the wall up-to two third in most of the area. Sections from the lower uterine segment revealed invasion of villi throughout the uterine wall and goes up-to serous coat. Proliferation of the muscle fibre and dilated blood vessels are noted.So histopathological diagnosis was Placenta Percreta.

\section{Discussion:}

The most rare and the most severe type of morbidly adherent placenta is the placenta percreta, where the placenta invades through the myometrial and the serosal layers. This can lead to the attachment of placenta to the nearby organs like the bowel,bladder.Placenta percreta may result in uterine rupture and life threatening haemorrhage. ${ }^{7}$ Placenta percreta,the rarest form of placentaladhesion seen in $5-7 \%$ of all abnormal placentation. ${ }^{7}$

Predisposing factors for placenta accrete are previous uterine damage due to prior uterine surgery leading to scarred uterus as caesarean section, myomectomy,uterine perforation and placenta praevia. Women with placenta praevia have high chances of morbid adhesion if placenta is anterior and they previously been delivered by caesarean section. There is a dose response relationship between morbidly adherent placenta and caesarean section, which shows an increased incidence with increasing number of prior caesarean section. Pathophysiology for this abnormal placental development involves deficient deciduas with absence of the fibrinoid layer limiting penetration of trophoblastic villi beyond deciduas into the myometrium. It leads to absence of physiological plane cleavage for placental separation after delivery 
and thus interfere with normal mechanism of placental detachment and myometrial contraction to arrest haemorrhage. ${ }^{8}$

Antenatal diagnosis require imaging using Colour Doppler ultrasonography and MRI to predict the occurrence of placenta accreta in high risk patients.Ultrasonogram findings include loss of the normal hypoechoic myometrial zone,thinning or disruption of the hyperechoic interface between the uterine serosa and the bladder, intraplacental vascular lacunae and loss of normal venous flow pattern of the peripheral margin. ${ }^{9}$

RCOG recommends routine USG scan of all patients at $20-23$ weeks to be specifically examined for placental localization, in which a low lying anterior placenta in patient with prior cesarean section should be meticulously followed up even in absence of haemorrhage. ${ }^{10}$

Ultrasound is quiet accurate to predict severe placenta accreta in at risk patients, however, less severe cases may not have any ultrasound findings. Most reliable sign of placenta accreta is the presence of irregular vascular space with arterial flow. ${ }^{11} \mathrm{MRI}$ can be used to diagnose placenta accreta when ultrasound scan is inconclusive or when placenta is posterior.Definite diagnosis of placenta accreta can be made only by histopathologial examination. ${ }^{9}$

Management depends on several factors which include the clinical presentation and the degree of placenta accreta and the involvement of adjacent structures. ${ }^{7}$ Placenta percreta were traditionally managed by caesarean hysterectomy particularly in case of rupture uterus due to placenta percreta as it is a life saving procedure ${ }^{11}$ However in patient diagnosed antenatally to have placenta percreta the plan can be made for conservative management if the patient wishes to maintain her fertility. ${ }^{11}$ Conservative approach involves leaving the placenta in situ,in part with or without administration of methotrexate has been advocated. Internal iliac arteries catheterization with bilateral uterine artery embolization have been adopted in patient who remain stable. ${ }^{12}$ Both hysterectomy and conservative management require reasonable amount of skill and experience, which may not be present in low resource settings. ${ }^{13}$

Recently,a conservative surgical technique called Triple P Procedure" which involves peri-operative placental localization and delivery of the fetus via transverse uterine incision above the upper border of placenta;pelvic devascularization; and placental nonseparation with myometrial excision and reconstruction of the uterine wall has been described as a safe and effective alternative to intentional retention of the placenta or peripartum hysterectomy . ${ }^{14}$

Attempt at conservative management should be mindful of the fact that the results are highly unpredictable, and the chance of subsequent successful pregnancy is also mediocre. ${ }^{13}$

\section{Conclusion:}

Perforating placenta percreata in a scarred uterus with history of previous caesarean section is a nightmare for obstetrician. Antenatal diagnosis by colour Doppler USG and MRI is essential for planning of safe delivery. Joint consultation of obstetrician, urologist and anaesthetist is essential for safety of the patient. Preoperative preparation with arrangement of blood transfusion is also an integral part of safe management. Incidental discovery during caesarean section bears life- threatening risk particularly in low resource set up where blood transfusion facility is not available. So accurate diagnosis of placental localization in patients with history of previous caesarean section is utmost important for safe obstetric management.

\section{References:}

1. Eller AG,Bennett MA,Sharshiner M,Masheter C,Soisson AP,Dodson M,et al .Maternal morbidity in cases of placenta accrete managed by a multidisciplinary care team compared with standard obstetric care.Obstet Gynecol 2011;117:331-7.

2. Demirci O, Tugrul AS, Yilmaz E, Tosun O, Demirci E, Eren YS. Emergency peripartum hysterectomy in a tertiary obstetric center: nice years evaluation. J. Obstet. Gyanecol. Res. 2011;37:1054-60.

3. ChenCH, Wang PH, Lin JY, Chiu YH, Wu HM, Liu WM.Uterine rupture secondary to placenta in a near -term pregnant woman with a history of hysterectomy.j Obstet Gynaecol Res 2011;37:71-4.

4. Aguilar-Hemandez OF, Renan-Rivero Y Coronado C,Sanchez- Garcia JF, Bolio-Bolio MA.Uterine rupture of placenta accrete.Ginecol Obstet Mex 2010;78:250-3. 
5. ACOG committee opinion.Placenta accreta.Number 266,January 2002.American College of Obstetricians and Gynaecologist.Int j Gynaecol Obstet 2002;77:77-8.

6. Comstock $\mathrm{CH}$.Antenatal diagnosis of placenta accrete:a review ultrasound Obstet Gynecol.2005;26(1):86-96.

7. Surabhi B,Ayanthi G,D Economide.A rare presentation of placenta percreta-intra-abdominal bleed and acute abdomen at 25 weeks of gestation:a case report.e-poster online:EP1.117,RCOG World Congress 2013.

8. Saima Aziz, Rubina Ather-Morbidly adherent placenta: can we limit the after math. Journal of the College of Physicians and Surgeons of Pakistan,2011;21(9):556-558

9. Derman AY,Nikac V,Haberman S,Zelenko N,Opsha O,Flyer M.MRI of placenta accrete:A new imaging perspective.AJR 2011;197:1541-21.
10. Royal College of Obstetrician and Gynaecology. Placenta praevia and placenta praevia accreta: diagnosis and management (Green-top 27) [Internet]. 2011. [updated 2011 Apr 11]. Available from: http://www.rcog.org.uk/ womens-health/ clinicalguidance/placentapraevia-and- placenta-praevia-accretadiagnosisand-management.

11 Sahera M.Case report:Placenta percreta and uterine rupture at 16 weeks. Saudi Medical journal 2013;34(7):753-756.

12. Clement D,Kayem G,Carol D.Conservative treatment of Placenta percreta:Safe alternative. EurreprodBiol 2004;114:108-109.

13. Lawrence Mbuagbaw et al:Per operative discovery of placenta praevia percreta:a case report.Pan African Medical Journal,2013,16:53.

14. Latika N, Edwin C.Management of morbidly adherent placenta.Journal of Obstetrics gynaecology and reproductive medicine. 2013 :23(7): 214-222. 